\section{LA CREACIÓN DE LA VENTAJA COMPETITIVA DESDE LA PERSPECTIVA DE LAS TEORÍAS ADMINISTRATIVAS*}

THE CREATION OF COMPETITIVE ADVANTAGE FROM THE PERSPECTIVE OF ADMINISTRATIVE THEORIES

\section{Cynthia Lizeth Martínez Aragón | Alejandro Arellano González | Blanca Carballo Mendívil}

\section{RESUMEN}

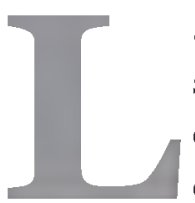

as organizaciones se encuentran en una lucha constante por subsistir ante un entorno complejo e inestable. La globalización ha permitido el incremento de empresas rivales que buscan posicionarse dentro del mercado. Para lograr el posicionamiento, se han visto en la necesidad de generar ventajas competitivas. Bajo este escenario, el presente artículo tiene el objetivo de exponer una perspectiva acerca de la ventaja competitiva desde diferentes teorías administrativas, mediante una revisión de literatura, presentando los principales resultados organizados por subtemas dentro de los cuales se encuentra: la teoría general de sistemas, teoría de recursos y capacidades, teoría del caos, relaciones interorganizacionales, administración estratégica, teoría basada en la gestión del conocimiento y ventaja competitiva. Se concluye que la capacidad de adaptación posibilitará a las empresas tener éxito en el mercado y por consecuencia, lograr rentabilidad, competitividad y no desaparecer.

Palabras clave: organización, ventaja competitiva, teorías de la administración, competitividad.

\begin{abstract}
Organizations are in a constant struggle for a complex and unstable environment. Globalization has allowed the increase of rival companies that seek to position themselves within the market. To achieve positioning, we have seen the need to generate competitive advantages. The present article has the objective of exposing a perspective about the competitive advantage of different administrative theories, through a literature review,

\footnotetext{
"Publicación financiada con recursos del Programa Fortalecimiento de la Calidad Educativa (PFCE-2019) de la Secretaría de Educación Pública (SEP) de México y del Programa de Fomento y Apoyo a Para la Investigación (PROFAPI-ITSON)
}

Cynthia Lizeth Martínez Aragón cynthiamrtz@hotmail.com

Alejandro Arellano González aarellanog@gmail.com

Blanca Carballo Mendívil bcarballom@gmail.com

Instituto Tecnológico de Sonora $M E ́ X I C O$

COMO CITAR ESTE ARTÍCULO

Martínez Aragón C. L., Arellano González A. y Carballo Mendívil B. (2020). La creación de la ventaja competitiva desde la perspectiva de las teorías administrativas. Revista de la Facultad de Ciencias Económicas, 24( 1), $79-92$ http://dx.doi.org/10.30972/rfce.2414362

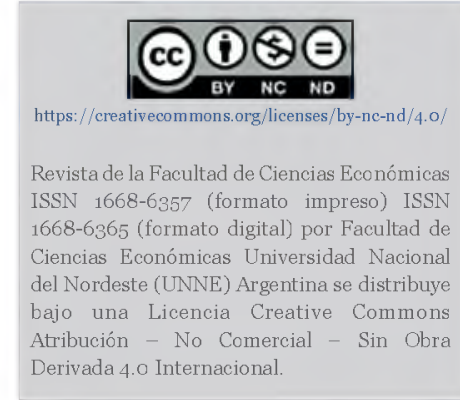


presenting the main results organized by subtopics within which is: the general theory of systems, theory of resources and capabilities, chaos theory, interorganizational relations, strategic management, theory based on knowledge management and competitive advantage. It is concluded that the ability to adapt will enable companies to succeed in the market and production, achieve profitability, competitiveness and not disappear.

Keywords: organization, competitive advantage, administration theories, competitiveness.

\section{INTRODUCCIÓN}

Las organizaciones se encuentran en una lucha constante por subsistir ante un entorno tan complejo e inestable. Además, la globalización ha permitido el incremento masivo de empresas rivales que buscan posicionarse dentro del mercado. Para lograr el posicionamiento, se han visto en la necesidad de generar ventajas competitivas, así como estudiar las formas de administrar e implementar buenas practicas que les permita adaptarse, al igual que mejorar sus procesos, servicios y productos, para generar la diferenciación y permanecer en el mercado (Gallegos, 2007).

Primeramente, la ventaja competitiva se conceptualiza como una diferenciación de la empresa que sea de valor para sus clientes. De igual manera, para alcanzar una ventaja, es necesaria la realización de acciones que permitan una mayor eficiencia, ya sea mediante el suministro de bienes y servicios igual o más eficiente que sus competidores, o bien, la habilidad de permanecer en el mercado (Alic, 1987; Tamames, 1988; Porter, 1990).

Dentro de los principales antecedentes, se parte desde la administración científica de Taylor, dónde se buscó reemplazar el empirismo por métodos científicos y actividades mecánicas (Taylor, 1983). Además, el lograr la especialización de los trabajadores, para la disminución de gastos de producción y generar una mayor eficiencia (Velásquez, 2002). Asimismo, con la aplicación de nuevas tecnologías en maquinarias y formas de trabajar, se buscaba mejorar los procesos de las empresas (Calderón, Magallon \& Núñez, 2010).

Posteriormente, Fayol, en su doctrina, propone los principios y elementos de administración que aseguran la gerencia, aludiendo que entre mayor sea el nivel jerárquico, la importancia de la capacidad administrativa aumenta, disminuyendo la capacidad técnica (Fayol, 1987). Lo cual, se consideró como base fundamental para el logro de objetivos, obteniendo mayores beneficios económicos y organizacionales. Según Ganga, Piñones y Valderrama (2014) Henri Fayol, estableció el proceso administrativo, enfocándose en la organización para generar una mejor eficiencia, en la cual hacia énfasis en la distribución y las funciones de altos mandos.

Bajo este escenario, el presente artículo tiene el objetivo de exponer una perspectiva acerca de la ventaja competitiva desde diferentes teorías administrativas, mediante una revisión 
de literatura acerca de la temática, manifestando los principales resultados organizados por subtemas para una mayor comprensión de los tópicos abordadas.

\section{MARCO DE LA INVESTIGACIÓN}

Para la realización de la presente investigación, se realizó una revisión de literatura, ya que tal como lo menciona Knopf (2006) eso permite obtener los siguientes beneficios: brindar una descripción general, identificar aspectos relevantes, brindar nuevas ideas y perspectivas, revelar problemas o áreas de oportunidad en la temática a abordar, finalmente, presentar nuevas conclusiones que permitan un contexto más amplio de investigación.

Para ello, se llevaron a cabo siete tareas propuestas por Fink (2013): 1) identificar la problemática a investigar, 2) selección de bases de datos y fuentes confiables, 3) selección de técnicas para la búsqueda de información, 4) criterios de evaluación, 5) criterios metodológicos para elegir artículos, 6) revisiones para validar artículos confiables, finalmente, 7) recapitulación de información. Las cuales se detallan a continuación.

Es decir, después de identificar las temáticas de estudio, se optó por realizar una búsqueda profunda de artículos científicos en bases de datos, tales como Emerald, Elsevier, EBSCO, google académico, entre otras, así como en revistas científicas y libros reconocidos como las principales obras de administración, para adquirir información fiable. Se optó por tomar también en consideración algunos artículos antiguos y clásicos, considerados como estudios seminales debido a que iniciaron el debate sobre algunos temas aquí abordados, es por ello que se consideraron aportaciones importantes de autores precursores (Bertalanffy, Fayol, Porter, etc), asimismo, se buscaron artículos más recientes para enriquecer y complementar la aportación. Se decidió buscar documentos en idioma inglés y español, dando importancia a artículos provenientes de revistas de alto impacto. Dentro de las palabras clave se utilizaron: ventaja competitiva, teorías de la administración, teoría de sistemas, competitividad, así como en idioma inglés: competitive advantage, management theories, general systems theory, competitiveness. Finalmente, se realizó un análisis para sintetizar los hallazgos, que se describen a lo largo del documento.

\section{RESULTADOS Y DISCUSIÓN}

\section{Teoría General de Sistemas}

Primeramente, un sistema es considerado como un conjunto de partes ordenadas que están interrelacionadas entre sí (Kats \& Rosenzweig, 1985; Bertalanffy, 1989). Se distingue por su forma 
sistemática y científica que pretende analizar la realidad (Arnold \& Osorio, 1998). Además, busca ser holística, es decir, considerar una parte específica o algo que sea perteneciente a un todo y para comprender el todo, se debe analizar cada una de sus partes (internas y externas) por separado, para generar soluciones o mejoras de problemas organizacionales (Bertoglio \& Johansen, 1982).

Los sistemas permiten definir y plantear de mejor forma las situaciones complejas para tomar las acciones administrativas adecuadas para las diferentes organizaciones, y evitar los problemas que se pudieran presentar por los cambios del entorno. Por otro lado, los sistemas brindan información importante para los administradores, para utilizarla en sus procedimientos, la información ayuda a los administradores a crear procesos y acciones que sean adecuadas, con el soporte de tecnologías y ambientales, las cuales, generan aspectos reales de planeación y control (Kast \& Rosenzweig, 1985).

Si bien, anteriormente, la organización era considerada como un sistema cerrado por su estructura totalmente definida. Sin embargo, con el paso del tiempo, los cambios tecnológicos y la modernidad, pasa a ser vista como un sistema abierto, por su interacción con el entorno, en las cuales se toman en cuenta cinco elementos: objetivos, valores, subsistemas técnico, estructural, psicosocial y administrativo. Los sistemas brindan información de importancia para administradores, permitiendo crear o adaptar buenas prácticas, que pudiesen generar ventajas diferenciadoras, ante la competencia.

\section{Teoría de recursos y capacidades}

Respecto al surgimiento de esta, comenzó a establecerse en los años 80's, tomando importancia debido a que buscaba describir, explicar y predecir las relaciones organizacionales. Para evitar el declive de las empresas, es necesario innovar, es decir, crear estrategias que permitan la supervivencia y hacer frente a la competencia (Barney, 2011). De igual manera, es posible clasificar los recursos en: recursos de capital físico (tecnología, equipo, estructura, materia prima), recurso de capital humano (capacitación, aprendizaje, conocimientos) y el recurso de capital organizacional (estructura y planificación de la empresa, relaciones formales e informales de la empresa). Sin embargo, se resalta que no necesariamente los tres recursos generan estrategias, incluso algunos pueden impedirla, dependiendo de su forma de ejecutarlos (Barney, 1991).

Ahora bien, acorde a Álvarez (2007) surge como opción para la dirección estratégica y generar ventajas competitivas, además, de la posibilidad de que sus procesos sean realizados de manera heterogénea dentro de las empresas que sean pertenecientes a un sector, o bien, sirvan de apoyo para lograr sus objetivos. Asimismo, el hacer hincapié en las capacidades y los recursos estratégicos, posibilitarán la obtención de mejores resultados, debido a que se llevan a cabo las actividades de manera eficiente, logrando mayores beneficios económicos.

Como se ha mencionado anteriormente, la globalización ha sido parte fundamental para que las empresas se amplíen o decidan restablecer sus actividades con mayor calidad, para satisfacer a sus consumidores, siendo la posición competitiva parte primordial para subsistir en el 
mercado, lo cual, debido a los cambios antes mencionados, resulta complicado, debido a que los clientes demandan mayor calidad, menores costos, productos y servicios amigables con el medio ambiente (Apodaca, Maldonado, \& Máynez, 2015; Quezada, 2004).

De igual manera, para generar ventajas competitivas sostenibles y hacer frente a la globalización, Vivas-López (2013) menciona que las organizaciones deberían optar por extender sus formas de llevar a cabo sus actividades, considerando los recursos intangibles han sido los que tomaron auge para la generación de éstas ventajas, es decir, los conocimientos que puedan generar valor tanto para los clientes como para las partes interesadas. Y con respecto a las estrategias, la teoría de recursos y capacidades es un complemento importante para la misma (Vivas-López, 2013; Gent \& Andalaft, 2007).

Complementando lo anterior, cada organización cuenta con sus recursos y capacidades, que los diferencian de las empresas que imitan ciertos procesos, lo cual, es posible obtener una ventaja competitiva, siendo así la competitividad como uno de los elementos fundamentales para lograr alcanzar, mantener y mejorar la posición dentro de un entorno tan turbulento y cambiante (García, 2006; Quezada, 2004). Las ventajas competitivas de una empresa, generalmente son debido al manejo óptimo y estratégico de sus propios recursos (Kung \& Wan, 2011).

\section{Teoría del caos}

Por otro lado, continuando con las teorías administrativas, según McBride (2005) la teoría del caos pretende estudiar el comportamiento inestable que se vive en los sistemas dinámicos, los cuales, no buscan ser lineales, ni deterministas. Además, pretende percibir la relación entre los sistemas de información con el entorno de las organizaciones. Lo complejo, inconsistente y no lineal, hace referencia a que las formas de comportarse de la organización no son las mismas, no se repiten y no son predecibles, por más parecida que sea una empresa, sus conductas no serán las mismas (McBride, 2005; Thietart \& Forgues, 1995).

Aunado a lo anterior, Thietart y Forgues (1995), aluden que la incoherencia y las actividades que se realizan sin ser planeadas, son unos de los principales factores para que una organización se encuentre inestable o desordenada, sin embargo, el desorden puede ser beneficioso, por dar apertura a nuevas formas de comportamiento, que en ocasiones podría facilitar la adaptación al ambiente, generando un mayor equilibrio, las organizaciones que experimentan son las que subsisten a los cambios.

Visto desde otra perspectiva, Thietart y Forgues (1995), mencionan que una organización ordenada, no siempre es positivo para las empresas, ya que el orden pudiera generar que los individuos no se preocupen por innovar o buscar alternativas que sean mejor, o bien, se resisten a los cambios, lo cual, les impide avanzar y por consecuencia desaparecer. La teoría de caos, se ha estudiado en diferentes puntos de vista, se ha aplicado ampliamente en campos financieros, predicción de precios de electricidad o energía, predicción de flujo de tráfico, entre otros. Asi como para describir a las organizaciones como sistemas complejos que se adaptan, para comprender 
y generar estrategias dentro de las empresas, o bien, para generar un cambio organizacional (McBride, 2005).

\section{Teoría de relaciones interorganizacionales}

Anteriormente, las teorías pretendían explicar ciertos fenómenos organizacionales, los investigadores buscaban principalmente la forma de aumentar o mejorar la producción mediante la eficiencia de manera sistemática, sin escuchar las ideas, necesidades o problemas que pudieran tener los obreros, lo cual, repercutía considerablemente en la manera de desempeñarse.

En ese contexto, surge la teoría de las relaciones interorganizacionales, que acorde a Provan y Milward (1995) es debido a que los investigadores notaron el aumento de redes organizacionales. Es decir, sin importar su giro o tipo de organización, en su mayoría buscaban la manera de relacionarse o cooperar entre sí para mejorar su competitividad o sus procesos, las organizaciones se dieron cuenta, que, creando estas conexiones, era más beneficioso para ellas.

Las organizaciones optan por aliarse para ser competitivas, lo cual, brinda beneficios a las partes interesadas por la colaboración y alianzas. Sin embargo, debe existir un compromiso y cumplirse, debido a que, si una organización falla o es ineficiente, puede afectar negativamente a las demás. Al momento de relacionarse y crear conexiones, beneficia la innovación, así como la agilización de sus procesos debido a que se identifican formas más adecuadas para llevarlas a cabo.

\section{Administración estratégica}

Tal como se ha mencionado, debido a los entornos turbulentos y a los cambios tecnológicos, las organizaciones necesitan estar a la vanguardia para generar ventajas competitivas, mediante procesos que las distingan, su posición en el mercado y la imitación de las buenas prácticas de la competencia. Lo cual, pudiera lograrse al planear estratégicamente, acorde a los objetivos de la organización (Teece, Pisano \& Shuen, 1997).

Primeramente, acorde a Mintzberg (1978) la estrategia se conceptualiza como una guía o un curso de acción, el cual está previamente planeado, para llevar a cabo una o varias situaciones específicas. Además, existen dos tipos de estrategias: las intencionadas y las realizadas, las cuales, sin importar a cuál se utilizará, resulta relevante tener conocimiento sobre las mismas, para llevarlas a cabo de la mejor manera posible.

Además, existen distintos puntos de vista sobre el pensamiento estratégico, De la Rosa, Barba y Montoya (2013) mencionan que, a pesar de las posturas, se tiene una influencia en aspectos sociales, económicos, de innovación y administrativos. Una organización que piensa estratégicamente, se refiere a que planea su futuro, considerando factores internos y externos importantes, para que mediante la innovación se generen ventajas competitivas, que permitan a la empresa posicionarse en un mercado tan cambiante. 
Es decir, el ambiente donde se desarrollan las organizaciones es volátil y cambiante, lo cual, ha creado una lucha constante por subsistir y ha logrado que la competencia de todos los sectores vaya cada vez en aumento, por lo cual, las organizaciones buscan adaptarse, Teece, Pisano y Shuen (1997) mencionan que las estrategias competitivas, por lo general se enfocan en buscar un mejor posicionamiento dentro del mercado, inclusive, tienen problemas estructurales (barreras de entrada) lo cual genera áreas de oportunidad para la competencia.

\section{Teoría basada en la gestión del conocimiento}

Las organizaciones con el paso del tiempo han logrado ser exitosas, según Nonaka y Takeuchi (1995) es debido a su creación de conocimiento dentro de la misma, es decir, el generar y compartir conocimiento, para después aplicarlo en productos, servicios o bien, en procesos de la empresa. Dicha habilidad, es un aspecto importante para la innovación de una empresa y generar una ventaja competitiva.

Aunado a lo anterior, Nonaka (2007) menciona que cuando los mercados cambian en lapsos cortos de tiempo y exista mayor número de competidores, sólo las empresas que opten por crear conocimiento y compartirlo con el demás personal, permitirá la innovación mediante la creación de nuevos productos e implementación de tecnologías novedosas. Así como la posibilidad de subsistir dentro de un mercado altamente preparado y competitivo.

Por otra parte, Nonaka y Takeuchi (1995) aluden que el conocimiento no es algo que se pueda observar, ya que hace referencia a las ideas subjetivas, valores, intuiciones, acciones y experiencia de las personas. El conocimiento, se puede dividir en dos partes: primeramente, el explícito, que hace referencia a lo que se puede procesar por medio de una computadora o alguna base de datos y en segundo lugar el conocimiento tácito, donde lo subjetivo dificulta procesar el conocimiento adquirido de manera sistemática, por su intuición.

Las empresas competitivas, suelen ver a la organización como un sistema abierto que está en relación con el exterior, además de tener una visión hacia el futuro, buscando adaptarse a los diferentes cambios que pudieran suceder. (Nonaka \& Takeuchi, 1995). Asimismo, se alude a que es principalmente por la creación de conocimiento y la innovación, es decir, el crear nuevos procesos, productos y servicios que sean de valor para sus clientes, las empresas adquieren ventajas que las diferencian de sus competidores.

\section{Ventaja competitiva}

Anteriormente, el paradigma tradicional de la organización industrial buscaba presentar un modelo sistemático para que los puestos directivos pudieran evaluar la competencia interna. La estrategia, era considerada como una manera que tenía una empresa para competir, tomando decisiones entorno a sus objetivos, productos, mercados, mercadotecnia, manufactura, etc. $Y$ surgió como respuesta a la necesidad de ayudar a los puestos directivos a transformar el caos en orden, así como lograr la ventaja competitiva (Porter, 1981). 
Hoy en día, las empresas han optado por la planeación estratégica para generar ventajas ante la competencia. Así como identificar las fuentes de la ventaja competitiva, se requiere de una inspección a fondo sobre las actividades que se realizan dentro de la empresa, para lo cual, Porter (2004) propone la cadena de valor como una herramienta para separar a la empresa en sus diferentes actividades para comprender su comportamiento, sus fuentes y las actividades detonantes para su diferenciación, considerando así, que cuando dichas actividades se hacen con menos costos y recursos que las demás empresas, se logra la ventaja competitiva.

Es posible representar una empresa con la cadena de valor, por la manera en la que se realizan sus actividades de forma individual, lo cual, manifiestan la estrategia en la que se basan. El valor, es lo que las personas están dispuestas a pagar por lo que se les está ofreciendo, se puede medir por ingresos, precios y los artículos posibles para vender, se dice que una empresa es rentable cuando su valor rebasa los costos de creación de su producto (Porter, 2004).

Por otro lado, Porter (1979) menciona que existen cinco fuerzas competitivas, que pueden variar según el sector, en primer lugar, se encuentra el poder de negociación de los compradores o clientes con organizaciones que se encargan de producir un producto o servicio, el poder de negociación de los proveedores o vendedores debido a que es donde la empresa consigue sus insumos para la elaboración de bienes. Posteriormente, se encuentra la amenaza de nuevos competidores entrantes, la amenaza de productos sustitutos que hace referencia a los productos que cumplen con la misma función y satisface la misma necesidad, finalmente, la rivalidad entre competidores, donde las empresas ofrecen descuentos o promociones, limitando la rentabilidad del sector.

\section{CONCLUSIONES}

Finalmente, es posible observar que las teorías administrativas (ver tabla 1) han estado en constante evolución, en busca de la mejora continua y la aplicación de acciones que los diferencie ante la competencia existente y de los competidores entrantes, ofreciendo a sus clientes productos y servicios que sean de valor, para ser una empresa capaz de hacer frente a las adversidades.

Tabla 1.

La ventaja competitiva a través de las teorías administrativas 
Teoría Administrativa

$\underline{\text { Autor(es) }}$
Propuesta respecto a la ventaia competitiva
Bertalanffy (1989), Arnold y Osorio (1998), Bertoglio y Johansen (1982), Kats y Rosenzweig (1985).
Ver a la organización como "un todo" que para estudiarla, se debe analizar cada una de sus partes por separado, para generar soluciones o mejoras.
Teoría de recursos y capacidades Barney (2011), Barney (1991), Álvarez (2007), Vivas-López (2013), Gent y Andalaft (2007), García (2006), Quezada (2004).

Teoría del caos
McBride (2005), Thietart \& Forgues, (1995).
Los recursos y las capacidades crean diferenciaciones en las empresas.

Procesos heterogéneos para generar ventajas competitivas.

Lo inestable, puede dar apertura a nuevas formas de comportamiento, que faciliten la adaptación al ambiente para subsistir a los cambios, para comprender y generar estrategias dentro de las empresas, $o$ bien, para generar un cambio organizacional.

\begin{tabular}{|c|c|c|}
\hline $\begin{array}{l}\text { Relaciones } \\
\text { interorganizacionales }\end{array}$ & Provan y Milward (1995). & $\begin{array}{c}\text { Relacionarse, formar alianzas } \\
\text { y cooperar entre sí, para ser } \\
\text { competitivas, beneficiando a las } \\
\text { empresas colaboradoras y a las } \\
\text { partes interesadas. }\end{array}$ \\
\hline
\end{tabular}

Administración estratégica

Teece, Pisano y Shuen (1997) Mintzberg (1978),

De la Rosa, Barba y Montoya (2013).

Las organizaciones que planean estratégicamente: planean su futuro, consideran factores internos y externos, para ser innovadoras y generar ventajas competitivas, que permitan a la empresa posicionarse.
Teoría basada en la gestión del conocimiento
Nonaka y Takeuchi (1995) Nonaka (2007)
Fomentar el conocimiento dentro de la organización para aplicarlo en productos, servicios y procesos, que mediante la innovación, generar una ventaja competitiva.

Nota. Elaboración propia con base en autores. 
Es difícil predecir el futuro en un entorno tan complejo, resulta importante que las organizaciones se adapten a los diferentes comportamientos aprovechando las oportunidades, para generar una ventaja competitiva. Si las empresas quieren subsistir, necesitan analizar y estudiar profundamente los aspectos relacionados con la competitividad, para poder hacer frente a los competidores o a los posibles entrantes.

Analizando lo anterior, es vital que las organizaciones realicen acciones acordes a sus necesidades, para adaptarse a los posibles escenarios aprovechando las oportunidades y minimizar las amenazas, logrando generar estrategias competitivas que les permita posicionarse y subsistir. Además, es necesario analizar el entorno y tomar en consideración que existen empresas que pueden ser competencia directa o indirecta sin importar el lugar donde se encuentren.

\section{REFERENCIAS BIBLIOGRÁFICAS}

Alic, J. A. (1987). Evaluating industrial competitiveness at the office of technology assessment. Technology in Society, 9(1), 1-17. DOI: 10.1016/0160791x(87)90027-3

Álvarez, S. E. (2007). Decisiones de hacer o comprar en el ámbito de los sistemas de información: una aproximación desde la teoría de recursos y capacidades. Cuadernos de Economía y Dirección de la Empresa, 10(31), 223-247. Recuperado de:

https://www.sciencedirect.com/science/article/pii/S1138575807700890

Apodaca, Del A. L. E., Maldonado, R. S. E., \& Máynez, G. A. I. (2015). La ventaja competitiva, desde la teoría de recursos y capacidades. Global Conference on Business \& Finance Proceedings 10(1).

Arnold, M. y Osorio F. (1998). Introducción a los conceptos básicos de la teoría general de sistemas. Cinta de Moebio. Revista de Epistemología de Ciencias Sociales, 1(3). Recuperado de: https://www.redalyc.org/pdf/101/10100306.pdf

Barney, J. B. (1991). Firm resources and sustained competitive advantage. Journal of management, 17(1), 99-120. DOI: https://doi.org/10.1177/014920639101700108

Barney, J. B., Ketchen, D. J., \& Wright, M. (2011). The Future of Resource-Based Theory: Revitalization or Decline? Journal of Management, 37(5), 1299-1315. doi: 10.1177/0149206310391805 
Bertalanffy, V. L. (1989). Teoría General de los Sistemas; fundamentos, desarrollo, aplicaciones (Séptima ed.). México, D.F.: Fondo de Cultura Económica.

Bertoglio, O. J., y Johansen, O. (1982). Introducción a la teoría general de sistemas. México: Editorial Limusa.

Calderón O. G., Magallon, D. T., \& Núñez H. R. (2010). A cien años de la administración científica. Análisis de las aportaciones de Taylor. Revista Gestión y estrategia, (38), 31-48. Recuperado de: http://gestionyestrategia.azc.uam.mx/index.php/rge/article/view/104/97

Cristofaro, M. (2019). I feel and think, therefore I am: An Affect-Cognitive Theory of management decisions. European Management Journal. DOI: https://doi.org/10.1016/j.emj.2019.09.003

De la Rosa, A., Barba, A., \& Montoya, F. M. (2013). Pensamiento estratégico: reflexiones, propuestas y evidencias empíricas. Administración y Organizaciones, 16(30), 5-17.

Fayol, H. (1987). Administración industrial y general. Buenos Aires: El ateneo.

Fink, A. (2013). Conducting research literature reviews: From the Internet to paper, 4 th edn. Los Ángeles: Sage Publications.

Gallegos, H. (2007). Sistema Kaizen en la administración. Innovaciones de Negocios, 4(1), 1-38. Recuperado de: http://eprints.uanl.mx/12468/1/A1.pdf

Ganga, F. C., Piñones, M. A., \& Valderrama, C. H. (2014). Innovaciones teóricas en administración: una sinóptica mirada diacrónica. Prisma Social: revista de investigación social, 1(12), 688-707.

García, C. S. (2006). Importancia estratégica de los activos intangibles. Un análisis desde la perspectiva de la teoría de recursos y capacidades. Administración y Organizaciones, 9(17), 93-115.

Gent F., K., \& Andalaft C., A. (2007). Extensión de los postulados de la teoría de los recursos y las capacidades de la firma a campos psicológicos. Revista Economía y Administración, 44(68), 3556. Recuperado de: http://www2.udec.cl/ rea/REVISTA\%2OPDF/Rer68/rea68art3.pdf

Kast, F., \& Rosenzweig, J. (1985). Adninistración en las Organizaciones. Enfoque de Sistemas y de Contingencia. México: McGraw-Hill. 
Knopf, J. W. (2006). Doing a Literature Review. PS: Political Science \& Politics, 39(01), 127132. DOI: $10.1017 / \mathrm{s} 1049096506060264$

Kung, W. J., \& Wan, C. H. (2011). Competitive advantage analysis and strategy formulation of airport city development-The case of Taiwan. Transport Policy, 18(1), 276-288.

McBride, N. (2005). Chaos theory as a model for interpreting information systems in organizations. Information Systems Journal, 15(3), 233-254.

Miao, Z., Le, S., Jing, J., \& Xinggao, L. (2019) A multi-scale prediction model based on empirical mode decomposition and chaos theory for industrial melt index prediction. Chemometrics and Intelligent Laboratory Systems 186(2019), 23-32.

Mintzberg, H. (1978). Patterns in strategy formation. Management science, 24(9), 934-948.

Monahan, K. (2018). How Behavioral Economics Influences Management Decision-Making. A New Paradigm. United States: Academic Press.

Nonaka, I. (2007). La empresa creadora de conocimiento. Harvard Business Review, 69 (6), 1-10.

Nonaka, I., \& Takeuchi, H. (1995). The Knowledge-Creating Company. United States, New York: Oxford University Press.

Porter, M. E (2004). Ventaja competitiva: creación y sostenimiento de un desempeño superior. Distrito Federal: Grupo Editorial Patria.

Porter, M. E. (1979). How competitive forces shape strategy. Harvard Business School Press, 57 (2), $137-145$.

Porter, M. E. (1981). The contributions of industrial organization to strategic management. Academy of management, 6 (4), 609-620.

Porter, M. E. (1990). Ventaja competitiva: creación y sostenimiento de un desempeño superior. Distrito Federal: Grupo Editorial Patria.

Provan, K. G., \& Milward, H. B. (1995). A Preliminary Theory of Interorganizational Network Effectiveness: A Connparative Study of Four Connmunity Mental Health Systems. Administrative Science Quarteriy, 4O(1), 1-33. DOI: 10.2307/2393698 
Quezada, F. Q. (2004). Análisis cualitativo de los recursos y capacidades de una empresa. Horizontes Empresariales, 3(1), 29-39.

Recuperado de: http://revistas.ubiobio.cl/index.php/HHEE/article/view/2073/1935

Tamames, R. (1988). Diccionario de economía. Madrid: Alianza.

Taylor, F. W. (1983). Principios de la Administración Científica. México: Herrero Hermanos.

Teece, D. J., Pisano, G., \& Shuen, A. (1997). Dynamic capabilities and strategic management. Strategic management journal, 18(7), 509-533. Recuperado de: https://onlinelibrary.wiley. com/doi/abs/10.1002/(SICI)1097-0266(199708)18:7\%3C509::AID-SMJ882\%3E3.0.CO;2-Z

Thietart, R. A., \& Forgues, B. (1995). Chaos theory and organization. Organization science, 6(1), 19-31. Recuperado de: https://www.researchgate.net/profile/Bernard_Forgues/publication/236685298_Chaos_Theory_and_Organization/links/odeec52de98b1282feoooooo.pdf

Velásquez V. F. (2002). Escuelas e interpretaciones del pensamiento administrativo. Estudios gerenciales, 18(83), 31-55. Recuperado de: http://www.scielo.org.co/scielo.php?script=sci_ arttext\&pid=So123-59232002000200002

Vivas-López, S. (2013). Implicaciones de las capacidades dinámicas para la competitividad y la innovación en el siglo XXI. Cuadernos de administración, 26(47), 119-140. Recuperado de: https://revistas.javeriana.edu.co/index.php/cuadernos_admon/article/view/7098

\section{CURRICULUM VITAE}

\section{Cynthia Lizeth Martínez Aragón}

Instituto Tecnológico de Sonora, México. Licenciada en Administración de Empresas Turísticas por el Instituto Tecnológico de Sonora, y estudiante de Maestría en Gestión Organizacional (PNPC).

\section{cynthiamrtz@hotmail.com}

ORCID: https://orcid.org/oooo-0002-5946-3873

\section{Alejandro Arellano González}

Instituto Tecnológico de Sonora, México. Ingeniero Industrial Mecánico con Maestría en Ingeniería en Optimización de Sistemas Productivos y Doctorado en Planeación Estratégica para 
la Mejora del Desempeño. Profesor investigador, Miembro del SNI (C), con perfil PRODEP y Líder del Cuerpo Académico Cadenas Productivas (Consolidado), en el departamento de Ingeniería Industrial en el Instituto Tecnológico de Sonora, México.

\section{aarellanog@gmail.com}

ORCID: https://orcid.org/oooo-00o2-6594-8391

\section{Blanca Carballo Mendívil}

Instituto Tecnológico de Sonora, México. Ingeniera Industrial y de Sistemas, con Maestría en Ingeniería en Logística y Calidad, Doctorado en Planeación Estratégica para la Mejora del Desempeño. Profesora investigadora auxiliar (depto. Ing. Industrial), Miembro del Sistema Nacional de Investigadores SNI (C).

bcarballom@gmail.com

ORCID: https://orcid.org/oooo-0003-0966-7146 